\title{
Effect of Entecavir on the Intestinal Microflora in Patients with Chronic Hepatitis B: A Controlled Cross-Sectional and Longitudinal Real-World Study
}

\author{
Yu-Xia Lu $\cdot$ Cheng-Zhi He $\cdot$ Ying-Xin Wang $\cdot$ Zi-Sheng Ai • \\ Ping Liang · Chang-Qing Yang
}

Received: July 28, 2020 / Accepted: October 8, 2020 / Published online: October 28, 2020

(C) The Author(s) 2020

\begin{abstract}
Introduction: This study aimed to analyze the diversity of intestinal flora in patients with chronic hepatitis B (CHB) and investigate the effect of entecavir on the intestinal flora in these patients.
\end{abstract}

Methods: Thirty patients with $\mathrm{CHB}$ and 30 healthy controls were recruited from the Department of Infectious Diseases and Department of Gastroenterology of Shanghai Tongji Hospital between January 2017 and December

Electronic supplementary material The online version of this article (https://doi.org/10.1007/s40121020-00355-w) contains supplementary material, which is available to authorized users.

Y.-X. Lu · C.-Z. He · Y.-X. Wang · C.-Q. Yang ( $\varangle)$ Department of Gastroenterology and Hepatology, Institution of Digestive Diseases, Tongji Hospital, Tongji University School of Medicine, Shanghai, China

e-mail: cqyang@tongji.edu.cn

Y.-X. Lu · C.-Z. He · Y.-X. Wang · C.-Q. Yang

Department of Infectious Diseases, Institution of Digestive Diseases, Tongji Hospital, Tongji

University School of Medicine, Shanghai, China

Z.-S. Ai

Department of Statistics, Tongji University School of Medicine, Shanghai, China

\section{P. Liang}

Department of Laboratory Medicine, Tongji

Hospital, Tongji University School of Medicine,

Shanghai, China
2018. Stool samples were collected for the detection of intestinal flora by high-throughput sequencing. Patients with $\mathrm{CHB}$ received antivirus therapy with entecavir for 8 weeks. The biochemical and virological responses were assessed and the intestinal flora were compared. Results: After entecavir treatment, the blood levels of alanine aminotransferase (ALT), interleukin-6 (IL-6), IL-8, tumor necrosis factor (TNF), and hepatitis B virus (HBV) DNA reduced significantly in patients with $\mathrm{CHB}$ and the species abundance of intestinal flora increased markedly. In patients with $\mathrm{CHB}$, the unique genera included Butyrivibrio, Phaseolus acutifolius, and Prevotellaceae NK3B31 group before treatment and Howardella, Candidatus Stoquefichus, Citrobacter, Dysgonomonas, Faecalicoccus, Methanobrevibacter, Mitsuokella, Mobilitalea, Succinivibrio, Gluconobacter, and Plesiomonas after treatment. The abundance of the following genera increased significantly after entecavir treatment in patients with CHB: Clostridium sensu stricto 1, Erysipelotrichaceae UCG-007, and Intestinibacter. The abundance of Streptococcus, Atopobium, and Murdochiella reduced markedly after entecavir treatment in patients with CHB. Conclusion: After 8-week entecavir treatment, the blood biochemical, immunological, and virological responses improved significantly, the species abundance of intestinal flora increased markedly, and there were unique genera in patients with $\mathrm{CHB}$ before and after treatment. 
Keywords: Chronic hepatitis B; Entecavir; Intestinal flora

\section{Key Summary Points}

The blood biochemistry, immunity, and viral load of patients with $\mathrm{CHB}$ were improved after entecavir treatment.

The healthy control group had the highest abundance of species of the intestinal flora, followed by the CHB group after entecavir treatment, and the patients with CHB before treatment had the lowest abundance of species.

The four bacterial families with significant difference before and after entecavir treatment were Caulobacteraceae, Methanobacteriaceae, Streptococcaceae, and Succinivibrionaceae.

\section{DIGITAL FEATURES}

This article is published with digital features, including a summary slide, to facilitate understanding of the article. To view digital features for this article go to https://doi.org/10.6084/ m9.figshare.13056197.

\section{INTRODUCTION}

Chronic hepatitis B (CHB) is a chronic progressive disease caused by hepatitis B virus (HBV) infection. Without timely and effective antiviral treatment, $\mathrm{CHB}$ will progress slowly and the risk of $\mathrm{CHB}$ eventually progressing into cirrhosis, liver failure, or hepatocellular carcinoma is about $15-40 \%$. Thus, CHB has been a worldwide public health burden. Anti-HBV treatment has been recognized as the key to the management of $\mathrm{CHB}[1,2]$. Increasing numbers of studies have shown that the pathogenesis of $\mathrm{CHB}$ is related to intestinal flora disorder. In this study, the diversity of intestinal flora was analyzed in patients with $\mathrm{CHB}$ who were treated with entecavir and in healthy controls, and the effects of entecavir on the intestinal flora were further investigated in patients with CHB. Our findings may provide evidence to aid the development of drugs targeting the intestinal flora in patients with CHB.

\section{METHODS}

\section{Subjects}

Han Chinese patients and controls were recruited into the present study. Stool samples were collected from 30 patients with $\mathrm{CHB}$ in the Department of Infectious Diseases and Department of Gastroenterology of Shanghai Tongji Hospital between January 2017 and December 2018 before and after treatment with entecavir. In addition, 30 healthy volunteers were recruited as controls. Approval was obtained from the ethics committee of the Tongji Hospital, Tongji University School of Medicine. The procedures used in this study adhere to the tenets of the Declaration of Helsinki and informed consent was signed by each patient.

\section{Inclusion Criteria}

A total of 30 patients with $\mathrm{CHB}$ in immune clearance phase and reactivation phase were included in the present study. Patients were aged 18-65 years; CHB was diagnosed according to the diagnostic criteria from the "Guideline for the Prevention and Treatment of Chronic Hepatitis" (updated in 2015) developed by the Hepatology Branch and the Infectious Diseases Branch of Chinese Medical Association [3]. According to the serum alanine aminotransferase (ALT), HBV DNA level, severity of liver disease, age, and family history, the risk for disease progression was evaluated. Patients who were eligible for the antiviral treatment were recruited into the present study.

\section{Exclusion and Inclusion Criteria}

Exclusion criteria were as follows: (1) Pregnant women and breast-feeding women; (2) patients with psychosis; (3) patients with a tumor; (4) patients with hepatic cirrhosis; (5) patients with 
other liver diseases or infectious diseases; (6) patients with concomitant severe diseases of the heart, lung, kidney, central nervous system, gastrointestinal tract, and other systems; (7) patients having prior antiviral treatment or being treated with antiviral drugs; (8) patients who used antibiotics and microecological drugs 1 month before study; (9) patients having poor compliance due to various reasons, or unable to cooperate with treatment and follow-up.

Inclusion criteria for healthy controls were as follows: subjects were aged 18-65 years old; results of routine physical examination were normal; virus carriers were excluded after clinical examinations; subjects were not treated with antibiotics or microecological drugs 1 month before study.

All the patients and controls were from Shanghai. There was no dietary change in the study period. The staples of the diet were rice and wheat. The supplementary food included vegetables, fruits, meat, fish, and shrimps. They did not take antibiotics or microecological drugs, had regular moderate excise, and had no dietary supplements and drugs affecting the gastrointestinal flora. There was no history of drinking or smoking.

In 30 patients with $\mathrm{CHB}$, venous blood was collected before and after treatment, and the liver function, cytokines (interleukin-6 (IL-6), IL-8, and tumor necrosis factor (TNF)), and HBV DNA were detected. The liver function was measured with an automatic biochemical analyzer (AU5800; Beckman Coulter). The normal range of ALT is 9-40 U/l. The contents of IL-6, IL-8, and TNF were measured with a chemiluminescence immunoassay system (Immulite1000; Siemens). Normally, the concentrations of IL-6, IL-8, and TNF are lower than $5.9 \mathrm{pg} / \mathrm{ml}, \quad 62 \mathrm{pg} / \mathrm{ml}$, and $8.1 \mathrm{pg} / \mathrm{ml}$, respectively. HBV DNA was detected with realtime fluorescence PCR in a thermal cycler (ABI 7300). The kit for HBV DNA detection was from Shanghai Zhijiang Biotech Co., Ltd, and the lower limit of HBV DNA is $100 \mathrm{IU} / \mathrm{ml}$. In addition, stool samples were collected from patients with CHB before and after treatment and from healthy controls for the detection of intestinal flora by high-throughput sequencing. FloraPrep $^{\mathrm{TM}}$ sampling tube and primers were from
Admera Health Medical Technology Co., Ltd (Suzhou, China).

\section{Treatment}

Patients with $\mathrm{CHB}$ received routine antiviral therapy for 8 weeks with entecavir $(0.5 \mathrm{mg}$, qd, oral) (Zhengda Tianqing Pharmaceutical Group Co., Ltd., No. H20100019, 0.5 mg/tablet).

\section{Stool Collection}

Stool samples were collected into FloraPrep ${ }^{\mathrm{TM}}$ tubes (Suzhou Admerahealth Medical Technology Co., Ltd.), stored at $4{ }^{\circ} \mathrm{C}$, and subjected to high-throughput sequencing within 2 weeks.

\section{Fecal Detection}

$16 \mathrm{~S}$ rDNA amplicon sequencing was done to detect the diversity of intestinal flora. In brief, DNA was extracted from the stool samples, followed quality assessment. The amplification of the $16 \mathrm{~S}$ rDNA variable region was done by PCR, and the products were purified. Then, the DNA library was established and detected, followed by high-throughput sequencing. After quality control, the volume of the DNA library was adjusted according to the target data, and several libraries were mixed for Illumina MiSeq sequencing.

\section{Statistical Analysis}

Statistical analysis was performed with SPSS version 20.0. The quantitative data with normal distribution are expressed as the means \pm standard deviation $(X \pm \mathrm{SD})$; the quantitative data with abnormal distribution are expressed as median and quartile (P25, P75). The gender was compared between two groups with the chisquare test. The differences in the parameters before and after treatment with normal distribution were compared with the $t$ test, and those with abnormal distribution with the Wilcoxon rank test. Data with normal distribution and homogeneity of variance were compared between two groups with the $t$ test and those with abnormal distribution and/or heterogeneity of variance were compared with the Mann-Whitney test. 


\section{RESULTS}

\section{General Characteristics}

There were 30 patients with $\mathrm{CHB}$ and 30 healthy controls in the present study. Of patients with $\mathrm{CHB}, 17$ were positive for $\mathrm{HBeAg}$ and 13 negative for HBeAg. They had no hepatic fibrosis, cirrhosis, and hepatocellular carcinoma. Table 1 shows the baseline characteristics of patients and controls. Results showed that there were no marked differences in the age and gender between patients with $\mathrm{CHB}$ and controls $(P>0.05)$.

\section{Blood Biochemical, Immunological, and Virological Responses After Treatment in Patients with CHB}

The liver function, cytokines (IL-6, IL-8, TNF), and HBV DNA level were detected before and after treatment in patients with CHB. As shown in Table 2, the blood levels of ALT, IL-6, IL-8, TNF, and HBV DNA reduced significantly after 8-week antiviral therapy in patients with $\mathrm{CHB}$ $(P<0.001)$.

\section{Changes of Intestinal Microflora in Patients with CHB After Entecavir Treatment}

After de-linking and low-quality filtering of the original data of each sequence were performed, the chimera sequences were removed and valid sequences were obtained for clustering analysis. Each cluster was assigned an operational taxonomic unit (OTU). At a similarity level of 97\%, all sequences were classified according to the OTUs, followed by bioinformatics analysis. On the basis of the OTU clustering analysis, the shared and unique OTUs were analyzed in patients with $\mathrm{CHB}$ before and after treatment and in healthy controls. A Venn diagram is shown in Fig. 1. Results showed that 600 OTUs were shared in patients with CHB before and after treatment and healthy controls. There were 20 unique OTUs in patients with $\mathrm{CHB}$ before treatment, 224 unique OTUs in patients with CHB after treatment, and 56 unique OTUs in healthy controls. This indicates that entecavir treatment increases the abundance of OTU (994) in patients with CHB.

The top 10 unique OTUs in patients with CHB before treatment were (high to low abundance) OTU413 (p_Bacteroidetes; c_Bacteroidia; o_Bacteroidales; f_Bacteroidales S24-7 group), OTU411 (p_Bacteroidetes; c_Bacteroidia; o_Bacteroidales; f_ Bacteroidaceae; g_Bacteroides), OTU92 (p_Firmicutes; c_Clostridia; o_ Clostridiales; f_Ruminococcaceae; g_[Eubacterium] coprostanoligenes group), OTU188 (p_Firmicutes; c_Clostridia; o_Clostridiales; f_Lachnospiraceae; g_ Butyrivibrio), OTU171 (Unassigned), OTU221 (p_Proteobacteria; c_Gammaproteobacteria; O_Pasteurellales; f_Pasteurellaceae; g_Haemophilus), OTU331 (p_Proteobacteria; c_Betaproteobacteria; o_Burkholderiales; f_ Alcaligenaceae; g_Sutterella), OTU314 (p_Firmicutes; c_Clostridia; o_ Clostridiales; f_Peptococcaceae; g_Peptococcus), OTU419 (p_Tenericutes; c_Mollicutes; O_NB1$\mathrm{n}$ ), and OTU350 (p_Bacteroidetes; c_Bacteroidia; o_Bacteroidales; f_Bacteroidaceae; g_Bacteroides).

The top 10 unique OTUs in patients with $\mathrm{CHB}$ after treatment were (high to low abundance) OTU893 (p_Actinobacteria; c_Actinobacteria; O_Bifidobacteriales; $\quad \mathrm{f}_{-}$ Bifidobacteriaceae; g_Bifidobacterium), OTU823 (p_Actinobacteria; c_ Actinobacteria; O_Bifidobacteriales; $\quad$ f_Bifidobacteriaceae;

Table 1 General characteristics of patients and controls

\begin{tabular}{lllll}
\hline Variables & CHB group $(\boldsymbol{n}=\mathbf{3 0})$ & Control group $(\boldsymbol{n}=\mathbf{3 0})$ & Statistics & $\boldsymbol{P}$ \\
\hline Gender $(\mathrm{M} / \mathrm{F})$ & $17 / 13$ & $18 / 12$ & 0.046 & 0.857 \\
Age $($ year $)$ & $42.30 \pm 11.35$ & $41.70 \pm 11.46$ & 0.113 & 0.884 \\
\hline
\end{tabular}


Table 2 Blood biochemical, immunological, and virological responses after treatment in patients with CHB

\begin{tabular}{lllrl}
\hline Variables & Before treatment $(\boldsymbol{n}=\mathbf{3 0})$ & After treatment $(\boldsymbol{n}=\mathbf{3 0})$ & Statistics & $\boldsymbol{P}$ \\
\hline ALT $(\mathrm{U} / \mathrm{l})$ & $211.00(152.00,319.00)$ & $90.50(57.25,125.25)$ & 7.927 & $<0.001$ \\
$\mathrm{IL}-6(\mathrm{pg} / \mathrm{ml})$ & $3.34(2.00,6.32)$ & $2.00(2.00,4.74)$ & -3.622 & $<0.001$ \\
$\mathrm{IL}-8(\mathrm{pg} / \mathrm{ml})$ & $45.62(13.21,132.55)$ & $22.23(10.46,57.30)$ & -4.453 & $<0.001$ \\
TNF $(\mathrm{pg} / \mathrm{ml})$ & $12.19(8.30,17.87)$ & $7.69(6.44,12.51)$ & -4.703 & $<0.001$ \\
HBV DNA $(\mathrm{IU} / \mathrm{ml})$ & $5.11 \times 10^{7}\left(5.09 \times 10^{6}\right.$, & $1.54 \times 10^{3}\left(1.00 \times 10^{2}\right.$, & -4.783 & $<0.001$ \\
& $\left.1.00 \times 10^{8}\right)$ & $\left.5.32 \times 10^{4}\right)$ & & \\
\hline
\end{tabular}

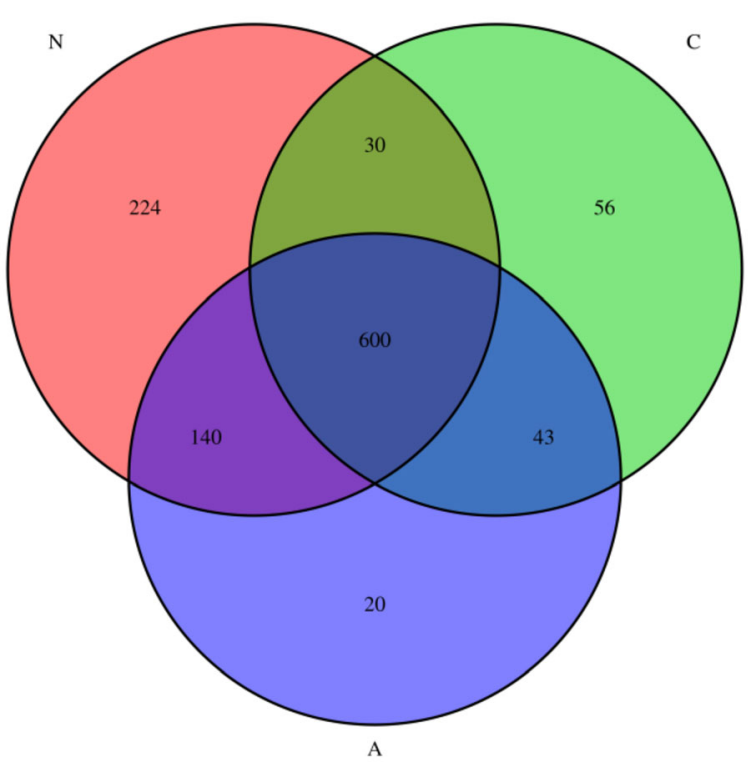

Fig. 1 Venn diagram of OTU in different groups (A: $\mathrm{CHB}$ patients before treatment; $\mathrm{N}$ : CHB patients after treatment; C: healthy controls)

g_Bifidobacterium), OTU963 (p_Firmicutes; c_Clostridia; o_Clostridiales; f_Clostridiaceae 1; g_Clostridium sensu stricto), OTU997 (p_Bacteroidetes; c_Bacteroidia; o_Bacteroidales; f_Prevotellaceae; g_Prevotella 9), OTU956 (p_Bacteroidetes; c_ Bacteroidia; o_Bacteroidales; f_Porphyromonadaceae; g_Parabacteroides), OTU962 (p_Bacteroidetes; c_Bacteroidia; O_Bacteroidales; f_Bacteroidaceae; g_Bacteroides), OTU793 (p_Bacteroidetes; c_Bacteroidia; o_Bacteroidales;
f_Rikenellaceae; g_Alistipes), OTU981 (p_Proteobacteria; c_Betaproteobacteria; o_Burkholderiales; f_Alcaligenaceae; g_Parasutterella), OTU1020 (p_Firmicutes; c_Clostridia; o_Clostridiales; f_Lachnospiraceae; g_Coprococcus 2), and OTU993 (p_Bacteroidetes; c_Bacteroidia; o_Bacteroidales; f_Prevotellaceae; $\mathrm{g}_{-}$ Alloprevotella).

The numbers of OTUs in different groups are shown in Table 3 and Fig. 2. Analysis of similarities (ANOSIM) showed that, although the abundance of species was different between groups, there was no marked difference in the intestinal flora between two groups before and after treatment $(P=0.422, R=0.002)$ (Table 3 , Fig. 2).

\section{Changes of Diversity of Intestinal Flora in Patients with CHB After Entecavir Treatment}

In order to understand the effect of nucleoside treatment on the diversity of intestinal microflora of patients with $\mathrm{CHB}$, stool samples were collected from patients with $\mathrm{CHB}$ before and after entecavir treatment, and from healthy controls. The Shannon index was used to analyze the diversity of intestinal flora in each sample (Fig. 3).

As shown in Fig. 3, the median Shannon index in patients with CHB was 4.668 before treatment and 4.819 after treatment, and it was 4.886 in healthy controls. This finding suggests that the abundance of intestinal flora is the highest in healthy controls, followed by the 
Table 3 OTU distribution in control group and CHB group

\begin{tabular}{llll}
\hline & Patients with CHB before treatment & Patients with CHB after treatment & Control group \\
\hline OTU (number) & $216.81 \pm 70.91$ & $204.04 \pm 9.72$ & $230 \pm 57.08$ \\
\hline
\end{tabular}

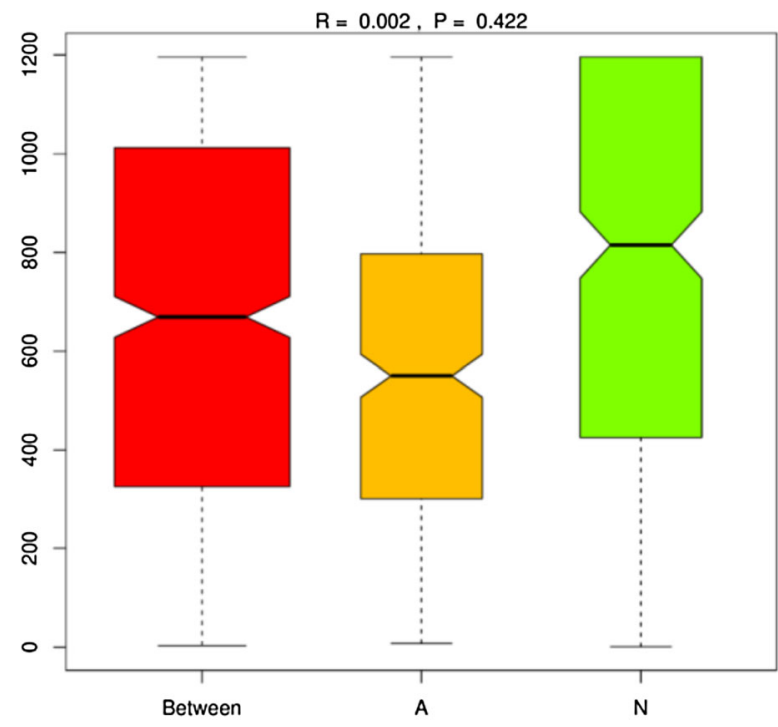

Fig. 2 ANOSIM of OTU in patients with CHB before and after treatment (A: $\mathrm{CHB}$ patients before treatment; $\mathrm{N}$ : $\mathrm{CHB}$ patients after treatment; C: healthy controls)

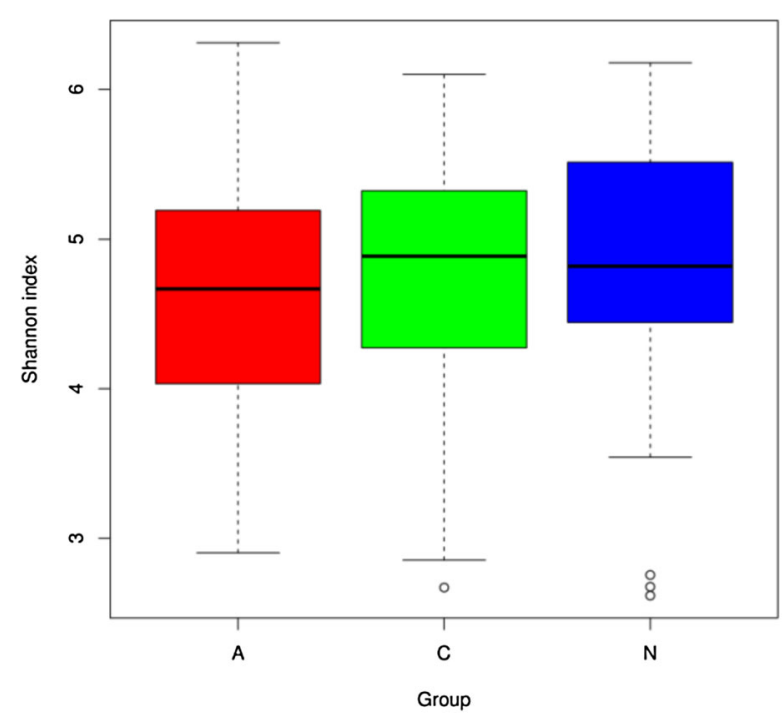

Fig. 3 Shannon index analysis of intestinal flora in different groups (A: CHB patients before treatment; N: $\mathrm{CHB}$ patients after treatment; C: healthy controls) patients with $\mathrm{CHB}$ after treatment, but it is the lowest in patients with $\mathrm{CHB}$ before treatment.

\section{Intestinal Microflora in Patients with CHB Before and After Entecavir Treatment}

In order to understand the effect of antiviral drug entecavir on the composition of intestinal flora in patients with $\mathrm{CHB}$, the intestinal flora was further analyzed at the level of genus in patients with $\mathrm{CHB}$ and healthy controls. The top 30 species were classified, the relative abundance of each sample was determined, and a histogram (Fig. 4) was drawn to display the proportions of different species in each sample. The abscissa shows the sample name, the ordinate shows the relative abundance of different species, the legend is the name of species at the genus level, and "Others" means the sum of relative abundance of all the species except for the top 30 species.

Further analysis showed that, at the genus level, the distribution of species was different between groups. In patients with $\mathrm{CHB}$ before treatment, the unique genera included Butyrivibrio, Phaseolus acutifolius, and Prevotellaceae NK3B31 group. After entecavir treatment, the unique genera included Howardella, Candidatus Stoquefichus, Citrobacter, Dysgonomonas, Faecalicoccus, Methanobrevibacter, Mitsuokella, Mobilitalea, Succinivibrio, Gluconobacter, and Plesiomonas. In patients with $\mathrm{CHB}$, the abundances of Clostridium sensu stricto 1, Erysipelotrichaceae UCG-007, and Intestinibacter after treatment were significantly higher than those before treatment $(P<0.05)$. In patients with $\mathrm{CHB}$, the abundances of Streptococcus, Atopobium, and Murdochiella before treatment were markedly higher than those after treatment $(P<0.05)$. 


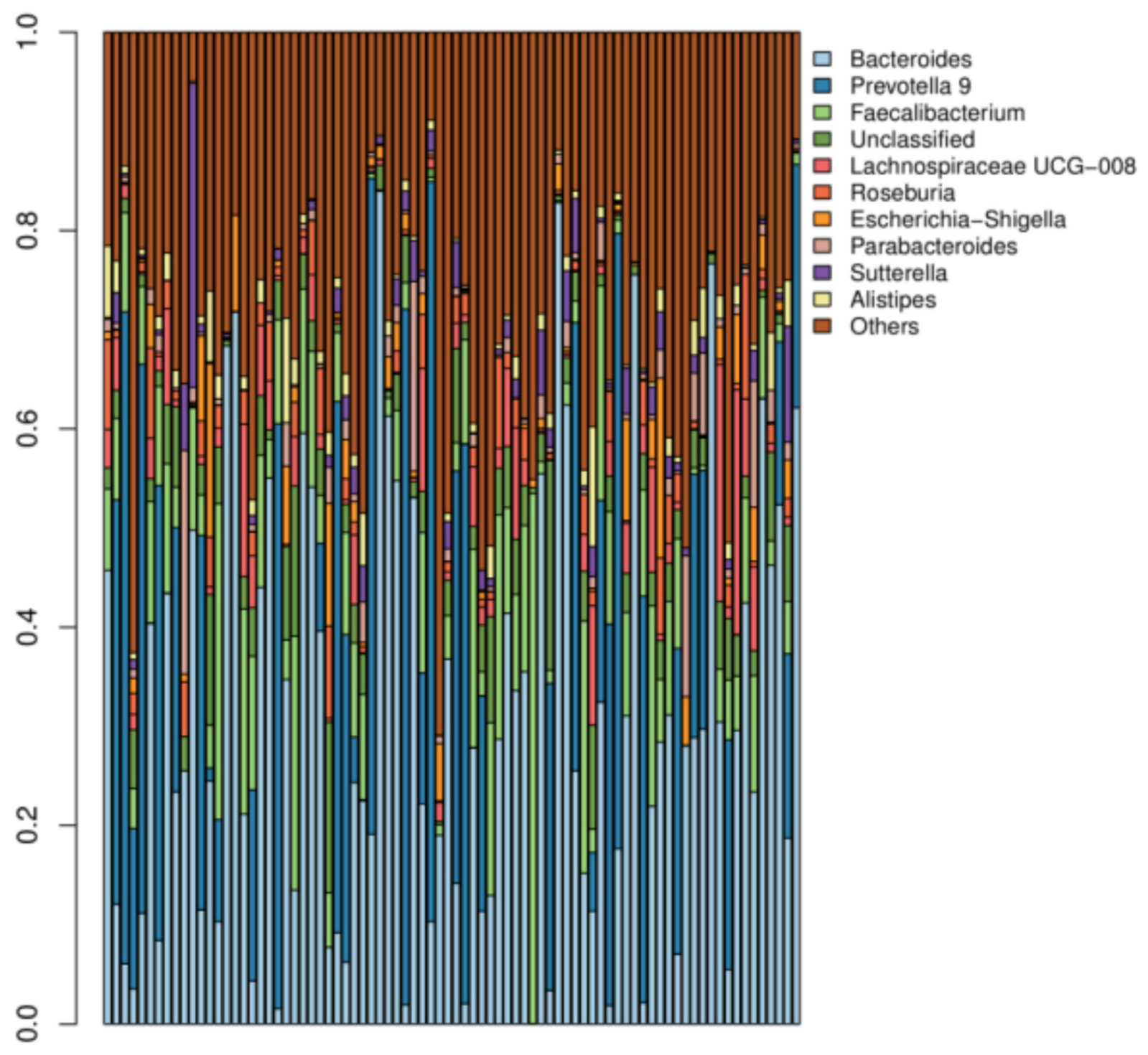

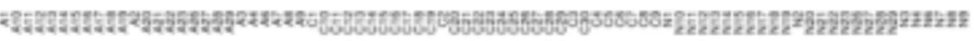

Fig. 4 Top 30 species with high abundance in different groups

\section{Different Intestinal Flora in Patients with CHB}

The intestinal flora was compared with Metastats analysis at family level in patients with $\mathrm{CHB}$ before and after treatment (Fig. 5). At family level, there were significant differences in the abundances of Caulobacteraceae, Methanobacteriaceae, Streptococcaceae, and Succinivibrionaceae in patients with CHB before and after treatment, and the abundance of
Streptococcaceae before treatment was dramatically higher than that after treatment.

\section{DISCUSSION}

The liver is the largest solid visceral organ in the human body. The intestine and liver share an embryonic origin-the foregut. The intestinal lymphocytes originate from the developing liver, and when they are mature, they may interact with the liver through the portal vein 


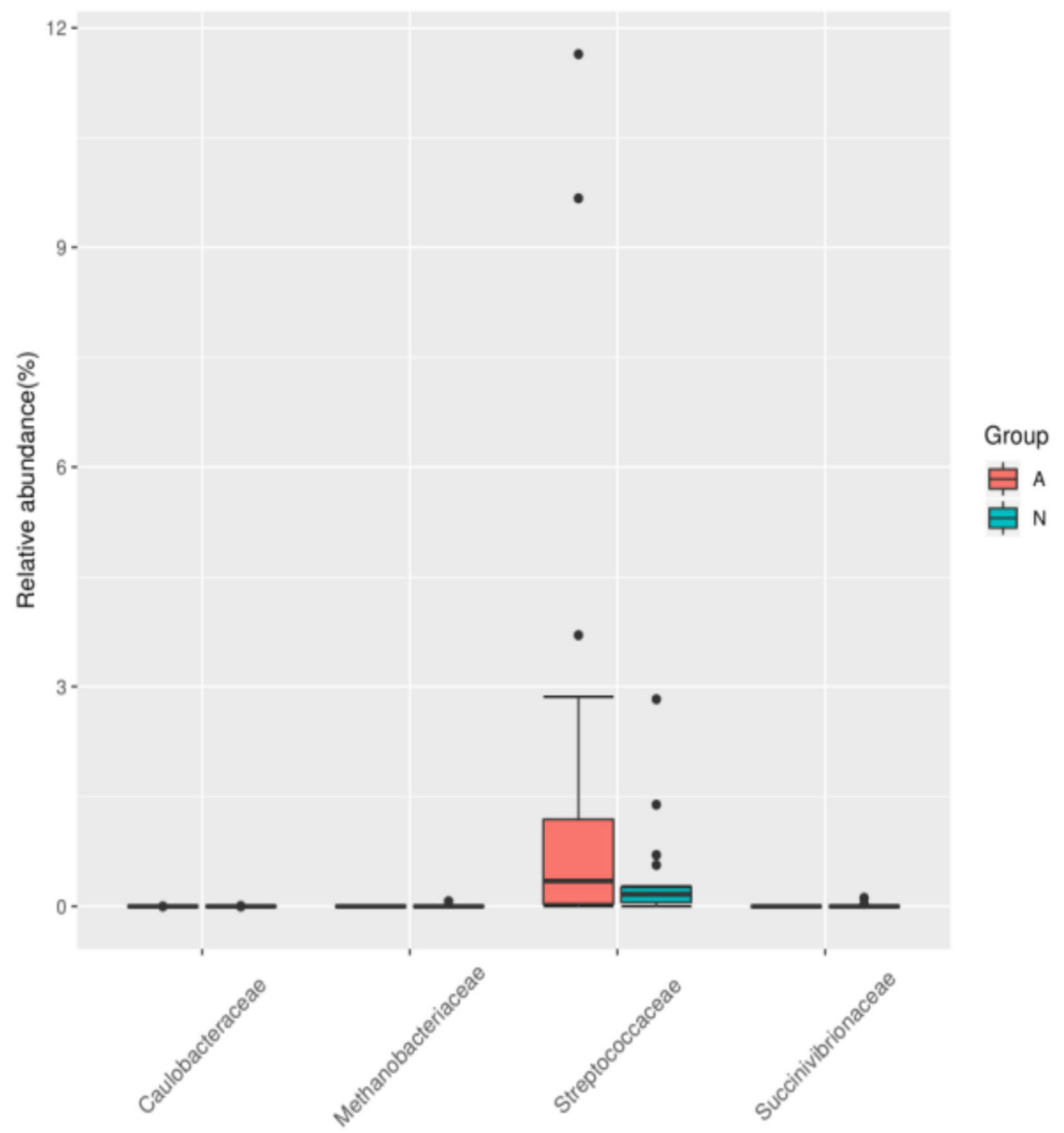

Fig. 5 Metastats analysis of intestinal flora in patients with $\mathrm{CHB}$ before and after treatment (A: CHB patients before treatment; $\mathrm{N}$ : CHB patients after treatment; C: healthy controls)

and biliary system. Almost all the blood leaving from the stomach and intestines will eventually enter the liver. A variety of studies have shown that the liver and intestine have a functional relationship at anatomical and biological levels. Marshall [4] proposed the concept of the "gut-liver axis" for the first time in 1998. The human gut contains a large amount of microorganisms, which play important roles in the regulation of metabolism and immunity of the host. Moreover, the intestinal flora also affects the development and physiology of the host. The latest literature has summarized the role of intestinal flora in the regulation of host physiological and pathological processes by focusing on the gut-liver axis [5, 6].

Some investigators [7] have evaluated the gut microbiota of patients infected with HBV. By using quantitative PCR and immunological techniques, studies have shown that Faecalibacterium prausnitzii, Enterococcus faecalis, Enterobacteriaceae, Bifidobacterium, and 
Bacterium acidi lactici (Lactobacillus, Pediococcus, Leuconostoc, and Weissella) in the intestine of patients with hepatic cirrhosis secondary to hepatitis B display significant variations. The ratio of Bifidobacterium to Enterobacteriaceae (B/ E) can reflect the colonization resistance of intestinal flora. This ratio significantly reduced from $1.15 \pm 0.11$ in healthy controls to $0.99 \pm 0.09$ in asymptomatic carriers, $0.76 \pm 0.08$ in patients with $\mathrm{CHB}$, and $0.64 \pm 0.09$ in decompensated hepatic cirrhosis $(P<0.01)$. This indicates that the $\mathrm{B} / \mathrm{E}$ ratio can reflect the imbalance of intestinal microecology during the progression of liver diseases. The levels of fecal SIgA and TNF in patients with decompensated hepatic cirrhosis secondary to hepatitis B were higher than in other groups, indicating that the human body attempts to achieve a new intestinal microecological balance through a complex self-regulating system.

The different clinical outcomes of patients with $\mathrm{CHB}$ are related to the interactions among virus, host, and environment, and are the result of multiple factors. Host factors include age on viral infection, gender, personal behaviors, genetic factors (gene polymorphism), and intestinal microecology [8]. The immune mechanism underlying the HBV initiating and maintaining acute or chronic infections (and even the hepatic cirrhosis and hepatocellular carcinoma) has not yet been determined. Increasing numbers of studies [9-15] have shown that the intestinal microecology is closely related to the occurrence and development of CHB. The intestinal flora can regulate the immune balance in the body, thereby protecting the immunity from the damage caused by hepatitis virus infection. The potential of intestinal flora to regulate the HBV infectionrelated immunity provides a new insight into the pathogenesis of HBV infection, and also presents a potential therapeutic target for HBV.

Antiviral therapy is a key component in the management of CHB. For patients with $\mathrm{CHB}$ receiving initial therapy with nucleoside (acid) drugs, current guidelines for the prevention and treatment of $\mathrm{CHB}$ recommend drugs (such as entecavir) with potent effectiveness and low resistance. Some studies [16-21] have shown that entecavir can effectively reduce HBV load, suppress liver inflammation, and help clear HBsAg and HBeAg, which may achieve functional cure of $\mathrm{CHB}$ and therefore inhibit the disease progression (such as hepatic cirrhosis and liver cancer). However, little is known about the effect of antiviral therapy with entecavir on the intestinal microecology of patients with CHB.

$\mathrm{Li}$ et al. [22] established a recombinant adeno-associated virus serotype 8 (rAAV8)-mediated continuous HBV infection mouse model. Results showed that entecavir could significantly reduce HBV DNA in the serum and liver. In further investigation, mice were divided into rAAV8-mediated CHB mice, the entecavir-treated CHB mice, and healthy controls. The 16S rDNA amplicon sequencing was done to detect the intestinal flora of the cecal contents, and the diversity of intestinal flora was analyzed. The results suggested that the alpha diversity of intestinal flora reduced significantly in rAAV8mediated CHB mice, and after 4-week entecavir treatment, the alpha diversity of intestinal flora increased markedly. In the rAAV8-mediated CHB mice, the abundances of Blautia and Clostridium spp. significantly decreased, and were negatively related to the HBsAg and HBeAg levels. On the contrary, the abundances of Butyricicoccus and Prevotellaceae NK3B31 were positively related to the $\mathrm{HBsAg}$ and $\mathrm{HBeAg}$ levels. In addition, the abundance of Akkermansia (known bacteria associated with intestinal barrier protection) significantly reduced in CHB mice, but returned to normal in the entecavir-treated mice and remained normal in the healthy control group. Moreover, the abundance of Akkermansia was negatively related to the serum and liver HBV DNA levels. This study shows that $\mathrm{CHB}$ mice have intestinal flora disorder, and entecavir can effectively reverse the intestinal flora disorder, revealing the role of intestinal flora in the $\mathrm{CHB}$ and antiviral therapy.

In the present study, patients with $\mathrm{CHB}$ and healthy controls were recruited, and patients with $\mathrm{CHB}$ were treated with antiviral entecavir for 8 weeks. Our results showed that the blood levels of ALT, IL-6, IL-8, TNF, and HBV DNA in patients with $\mathrm{CHB}$ reduced dramatically after antiviral treatment $(P<0.001)$. It indicates that 
the blood biochemical, immunological, and virological responses are significantly improved in patients with $\mathrm{CHB}$ after entecavir treatment.

Furthermore, 16S rDNA amplicon sequencing technology was performed for highthroughput sequencing of fecal samples from patients with $\mathrm{CHB}$ and healthy controls. Our results showed that patients with $\mathrm{CHB}$ had 20 unique OTUs before treatment, patients with CHB had 224 unique OTUs after treatment, and controls had 56 unique OTUs. This indicates that entecavir treatment increases the OTUs of intestinal flora of patients with CHB. ANOSIM showed that, although the abundance of species was different among groups, there was no significant difference in the intestinal flora of patients with $\mathrm{CHB}$ before and after treatment and between two groups $(P=0.422, R=0.002)$. Shannon index analysis showed that the abundance of species was the highest in the healthy controls, followed by the patients with $\mathrm{CHB}$ after entecavir treatment, and the abundance of species was the lowest in patients with $\mathrm{CHB}$ before treatment. There were unique OTUs in different groups and there was significant difference in the intestinal flora at different levels among groups $(P<0.05)$. The abundances of Caulobacteraceae, Methanobacteriaceae, Streptococcaceae, and Succinivibrionaceae were significantly different, and the abundance of Streptococcaceae in patients with CHB reduced markedly after treatment. Our study suggests that entecavir not only inhibits the replication of HBV but also regulates the intestinal microecology. The antiviral therapy may alter the intestinal flora via the following ways: antiviral therapy may reduce the HBV load and inhibit the expression of related antigens, which may further change the types and number of immune cells in the hepatic microenvironment; the antigens from intestinal flora may enter the circulation via enterohepatic circulation and interact with immune cells in the liver. Thus, antiviral therapy may indirectly affect the immune microenvironment in the liver to affect the intestinal flora. More studies with large sample size and long-term follow-up are needed to confirm our findings. This was also a limitation in the present study.
Currently, $\mathrm{CHB}$ is difficult to cure and requires long-term antiviral therapy and followup. A variety of studies have shown that the occurrence and development of CHB are closely related to changes in the intestinal microecology. Studies on the interaction of $\mathrm{CHB}$ and antiviral drugs with intestinal microecology will provide potentially effective targets for the functional cure of $\mathrm{CHB}$.

\section{CONCLUSION}

After 8 weeks of antiviral treatment with entecavir, the serum parameters related to biochemistry, immunity, and virology improved in patients with CHB. The intestinal flora had the highest abundance in species in the healthy control group, followed by the CHB group after entecavir treatment, and the patients with $\mathrm{CHB}$ had the worst abundance in the intestinal flora before treatment. In addition, the intestinal flora in patients with $\mathrm{CHB}$ before and after entecavir treatment had its own unique bacterial species and different bacteria.

\section{ACKNOWLEDGEMENTS}

We thank the participants of the study.

Funding. This study was supported by the National Natural Science Foundation of China (No. 81670571 and No. 81820108006) and Shanghai Key Clinical Specialty Construction Project (No. shslczdzk06801). The Rapid Service Fees were funded by the authors.

Authorship. All authors meet the International Committee of Medical Journal Editors (ICMJE) criteria for authorship for this article, take responsibility for the integrity of the work as a whole, and have given their approval for this version to be published.

Disclosures. Yu-Xia Lu, Cheng-Zhi He, YingXin Wang, Zi-Sheng Ai, Ping Liang, and ChangQing Yang declare that they have no conflict of interest. 
Compliance with Ethics Guidelines. Approval was obtained from the ethics committee of the Tongji Hospital, Tongji University School of Medicine. The procedures used in this study adhere to the tenets of the Declaration of Helsinki and informed consent was signed by each patient.

Data Availability. The datasets generated during and analyzed during the current study are available from the corresponding author on reasonable request.

Open Access. This article is licensed under a Creative Commons Attribution-NonCommercial 4.0 International License, which permits any non-commercial use, sharing, adaptation, distribution and reproduction in any medium or format, as long as you give appropriate credit to the original author(s) and the source, provide a link to the Creative Commons licence, and indicate if changes were made. The images or other third party material in this article are included in the article's Creative Commons licence, unless indicated otherwise in a credit line to the material. If material is not included in the article's Creative Commons licence and your intended use is not permitted by statutory regulation or exceeds the permitted use, you will need to obtain permission directly from the copyright holder. To view a copy of this licence, visit http://creativecommons.org/licenses/bync/4.0/.

\section{REFERENCES}

1. Razavi-Shearer D, Gamkrelidze I, Nguyen MH, et al. Global prevalence, treatment, and prevention of hepatitis B virus infection in 2016: a modelling study. Lancet Gastroenterol Hepatol. 2018;3: 383-403.

2. Ott JJ, Stevens GA, Groeger J, Wiersma ST. Global epidemiology of hepatitis B virus infection: new estimates of age-specific HBsAg seroprevalence and endemicity. Vaccine. 2012;30:2212-9.

3. Hou J, Wang G, Wang F, et al. Guideline of prevention and treatment for chronic hepatitis B (2015 update). J Clin Transl Hepatol. 2017;5:297-318.
4. Marshall JC. The gut as a potential trigger of exercise-induced inflammatory responses. Can J Physiol Pharmacol. 1998;76:479-84.

5. Fu ZD, Cui JY. Remote sensing between liver and intestine: importance of microbial metabolites. Curr Pharmacol Rep. 2017;3:101-13.

6. Koh JC, Loo WM, Goh KL, et al. Asian consensus on the relationship between obesity and gastrointestinal and liver diseases. J Gastroenterol Hepatol. 2016;31:1405-13.

7. Lu H, Wu Z, Xu W, Yang J, Chen Y, Li L. Intestinal microbiota was assessed in cirrhotic patients with hepatitis B virus infection. Intestinal microbiota of HBV cirrhotic patients. Microb Ecol. 2011;61: 693-703.

8. Xing HC. Host factors influencing the clinical outcome of chronic hepatitis B virus infection. Zhonghua Gan Zang Bing Za Zhi = Chin J Hepatol. 2017;25:495-9.

9. Xu D, Huang Y, Wang J. Gut microbiota modulate the immune effect against hepatitis $B$ virus infection. Eur J Clin Microbiol Infect Dis. 2015;34: 2139-47.

10. Derovs A, Laivacuma S, Krumina A. Targeting microbiota: what do we know about it at present? Medicina (Kaunas). 2019;55:459.

11. Milosevic I, Vujovic A, Barac A, et al. Gut-liver axis, gut microbiota, and its modulation in the management of liver diseases: a review of the literature. Int J Mol Sci. 2019;20:395.

12. Yang $\mathrm{R}, \mathrm{Xu} \mathrm{Y}$, Dai $\mathrm{Z}$, Lin $\mathrm{X}$, Wang $\mathrm{H}$. The immunologic role of gut microbiota in patients with chronic HBV infection. J Immunol Res. 2018;2018:2361963.

13. Tsai KN, Kuo CF, Ou JJ. Mechanisms of hepatitis B virus persistence. Trends Microbiol. 2018;26:33-42.

14. Schwenger KJ, Clermont-Dejean N, Allard JP. The role of the gut microbiome in chronic liver disease: the clinical evidence revised. JHEP Rep. 2019;1: 214-26.

15. Woodhouse CA, Patel VC, Singanayagam A, Shawcross DL. Review article: the gut microbiome as a therapeutic target in the pathogenesis and treatment of chronic liver disease. Aliment Pharmacol Ther. 2018;47:192-202.

16. Manne V, Gochanour E, Kowdley KV. Current perspectives into the evaluation and management of hepatitis B: a review. Hepatobiliary Surg Nutr. 2019;8:361-9. 
17. Mak LY, Seto WK, Fung J, Yuen MF. New biomarkers of chronic hepatitis B. Gut Liver. 2019;13: 589-95.

18. Wang $\mathrm{HW}$, Lai $\mathrm{HC}, \mathrm{Hu} \mathrm{TH}$, et al. On-treatment changes in FIB-4 and 1-year FIB-4 values help identify patients with chronic hepatitis $B$ receiving entecavir therapy who have the lowest risk of hepatocellular carcinoma. Cancers (Basel). 2020;12: 1177.

19. Cho JY, Sohn W, Paik YH, et al. Long-term hepatitis B surface antigen (HBsAg) kinetics during entecavir treatment in Korean patients-functional cure unlikely. J Viral Hepat. 2020;27(9):951-4.
20. Geng J, Bao H, Chen $Y$, et al. Nucleos(t)ide analogues for the treatment of chronic hepatitis B: a systematic review with network meta-analysis. Expert Rev Anti Infect Ther. 2020;18(8):823-34.

21. Wani MA, Sodhi JS, Zargar SA, et al. Entecavir is safe and effective in long term for the treatment of hepatitis B in immunocompromised children. J Clin Exp Hepatol. 2020;10:150-4.

22. Li X, Wu S, Du Y, Yang L, Li Y, Hong B. Entecavir therapy reverses gut microbiota dysbiosis induced by hepatitis B virus infection in a mouse model. Int J Antimicrob Agents. 2020;56:106000. 